\title{
The Role of Enterprise Architecture for Digital Transformations
}

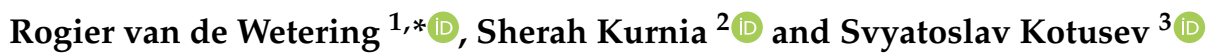 \\ 1 Department of Information Sciences, Open University the Netherlands, 6419 AT Heerlen, The Netherlands \\ 2 Computing and Information Systems, Melbourne School of Engineering, The University of Melbourne, \\ Parkville, VIC 3052, Australia; sherahk@unimelb.edu.au \\ 3 Higher School of Economics, National Research University, 101000 Moscow, Russia; kotusev@kotusev.com \\ * Correspondence: rogier.vandewetering@ou.nl; Tel.: +31-638-395-232
}

check for updates

Citation: van de Wetering, R.; Kurnia, S.; Kotusev, S. The Role of Enterprise Architecture for Digital Transformations. Sustainability 2021, 13, 2237. https://doi.org/10.3390/ su13042237

Received: 19 January 2021

Accepted: 17 February 2021

Published: 19 February 2021

Publisher's Note: MDPI stays neutral with regard to jurisdictional claims in published maps and institutional affiliations.

Copyright: (C) 2021 by the authors. Licensee MDPI, Basel, Switzerland. This article is an open access article distributed under the terms and conditions of the Creative Commons Attribution (CC BY) license (https:/ / creativecommons.org/licenses/by/ $4.0 /)$.

\section{Introduction}

In the current turbulent and unpredictable markets, competitive advantage can no longer be achieved through high product quality or efficient processes alone. Modern firms are currently pressured to increase both the efficiency and effectiveness of business operations to achieve the firm's strategic direction and unlock the true potential of new and disruptive digital business models [1,2]. Under these conditions, firms should align and integrate their information systems (IS), information technology (IT) assets, and resources with business processes to efficaciously respond to changing environmental and market conditions [3,4]. To orchestrate these business and IT assets and resources, components, and capabilities in digital and organizational transformations, firms increasingly adopt Enterprise Architecture (EA).

EA supports firms in the process of documenting current and desired future states of firms' digital infrastructure, processes, and capabilities [5,6]. EA, therefore, can be considered 'a strategy' to achieve specific business goals and objectives [5] and enable firms to become agile, aligned, deliver high-quality services [7], customer-focused, and enable better decision-making across the firm $[8,9]$. EA is regarded as crucial in designing firms for the digital era [10] and driving organizational change [11]. Our working definition for EA in this editorial is straightforward: a blueprint of firms in the business ecosystem that documents the current and desirable future states of firms' strategic goals, collaborative efforts, business processes, and associated IT landscape.

The strategic role of enterprise architecture (EA) in modern firms has gained considerable interest in the IS, management, and business and consulting communities. Over the past years, there have been numerous critical appraisals on the value and role of EA. However, in recent years, what we see in business is that firms have undergone a series of business and IT transformations (e.g., migration to the cloud, the introduction of analytics and AI tools, rationalization of legacy). EA maps out and orchestrates business and IT assets and processes and their interconnections and is, therefore, a crucial ingredient for firms to drive digital transformation. A quick search on Google at the end of December 2020 for the key term "Enterprise Architecture" yielded 9.1 million results. According to Gartner's Hype Cycle for Enterprise Architecture [12], EA has become even more crucial given the recent COVID-19 outbreak because firms are operating in highly turbulent business and technology environments. Hence, there is a need to support executives and decision-makers in making smarter decisions concerning the radically changing business and IT landscape as part of the digital transformation. Under these pressing circumstances, EA enables modern firms to actively respond to a wealth of disruptive and emerging forces by systematically sensing, identifying, and subsequently analyzing desired business outcomes [6,13-15].

We argue that EA is on the rise again, and research on the role of EA in digital transformations is very relevant for scholars and practitioners. However, what does this all mean for IS and EA researchers? Accordingly, we invited recognized scholars and 
practitioners within the EA domain to actively participate in this discussion by providing their perspective on what this value-creating role of EA should or can be. Hence, we welcomed a wide variety of original and high-quality EA papers, including conceptual and empirically validated work for this special issue. The editors embraced core criteria that each particular paper should push the EA domains frontiers, drive best practices and further thinking in this domain, and advance our understanding of EA in the age of digital transformation. In this editorial, our goal is to synthesize the existing body of knowledge and uncover some core assumptions related to how EA can guide modern firms' digital transformations.

The editorial is structured as follows. First, we highlight the key contributions of the five articles published in this special issue. We end this editorial with some concluding remarks.

\section{Papers in This Special Issue}

The carefully selected papers in this special issue are original, and each provides a unique insight into the domain of EA. The articles substantially extend the extant literature and push the EA domain's frontiers and advance our understanding of EA in the age of digital transformation. Each paper provides an innovative and rich perspective on the challenges that firms face with EA-enabled digital transformation, and they offer various theoretical and practical recommendations.

The included papers collectively offer the scholars an overview of EA work's range and reach and the novel use of EA in digital transformation. The five papers collectively investigate EA's role in digital transformations and the EA value-creating process within firms considering the business ecosystem. The papers also examine how to build sustainable IS, orchestrate EA resources, develop dynamic capabilities, and govern EA's business value realization process.

In 'Enterprise Architecture as Explanatory Information Systems Theory for Understanding Small-and Medium-Sized Enterprise Growth,' the authors particularly focus on small- and medium-sized enterprises (SME) as they play a significant role in solving socio-economic challenges. They claim that EA is crucial to understand and explain how firms operate and that we fundamentally need to revisit some EA applications. The first paper of this special issue applies IS theory perspectives and adopted the taxonomy and structural components of theory to argue that EA, as represented by the Zachman Framework for Enterprise Architecture (ZFEA), could be adopted as an explanatory IS theory. The paper adopts a multi-case approach to investigate whether different focus patterns could be detected during SME growth. The authors found support for their claim that EA, represented through an appropriate framework like the ZFEA, can serve as an explanatory theory for SMEs during start-up, growth, and transformation.

In 'Enterprise Architecture and Organizational Benefits: A Case Study,' the authors focus on identifying organizational benefits that can be achieved through EA implementation. They argue that despite the importance of existing EA frameworks, the literature still lacks support in realizing the potential benefits EA can create in organizations. Accordingly, this study extracted EA benefits from the literature, EA experts reconfirm benefits, and finally, validates an EA benefits model through a case study. The benefits considered in this study are related to EA products, services, and strategies are known as: (i) business agility, (ii) creating competitive advantage, and (iii) increasing value. This paper unfolds the steps and guidelines for assessing EA organizational benefits using corresponding measuring and sub-criteria. The authors suggest that firms can achieve benefits from the EA framework implementation, and the framework can likewise assist EA decision-makers in understanding and realizing the EA benefits in practice.

The next paper, 'Enterprise Architecture: A Business Value Realization Model,' contends that a more profound exploration of the dimensions of EA benefit realizations is required in the study of EA value realization. The author argues that the current absence of an EA benefit realization process model restrains the development of a clear business case for an EA because of the ambiguous justifications of EA's business value. The paper follows 
a process theoretical perspective to connect the dimensions that support the description and analysis of the EA benefit realization process. Outcomes of a case study to explore how EA investment is converted into organizational value showcase that the process model links and unpacks the EA value process into three iterative and interrelated processes: the EA conversion process, the EA use process, and the EA competitive process. This model provides an analytical lens to comprehend why EA funding does not always improve organizational performance from a managerial perspective. This work also identifies various factors that influence EA business value realization, including management support, communication and collaboration, EA team capability, EA framework and tools, EA vision, and EA governance.

The next paper, titled 'Development of Transportation Management System with the Use of Ontological and Architectural Approaches to Ensure Trucking Reliability,' specifically focus on the role of EA in the transport and logistics industry. The authors argue that the deployment and introduction of many current information systems and applications for motor transport and transport logistics solutions do not provide the expected results and business value. Moreover, the COVID-19 pandemic has sparked a surge in online commerce, which has prompted trucking companies to explore new ideas to meet the increased consumer demand. The authors argue that there is a need to directly describe the semantics of enterprise business unit interaction before the modeling stage. Hence, this paper proposes using an ontological approach to form these semantic relations and use the Zachman Framework to systematize the enterprise ontology and help assemble the "elementary particles" of the firm. Despite being excessively "technocratic" and formalized, the authors believe that combining this framework and enterprise ontology allows getting a consistent plan or a roadmap for realizing an IS implementation project.

Finally, 'The Effect of Enterprise Architecture Deployment Practices on Organizational Benefits: A Dynamic Capability Perspective,' focuses on EA-based capabilities that organize and deploy organization-specific resources to align strategic objectives with the technology's particular use. The authors argue that there is no conclusive evidence of how EA-based capabilities enable business transformations and deliver organizational benefits in the ever-changing economic environment. This paper develops and tests a new research model that explains how dynamic enterprise architecture capabilities lead to organizational benefits. This study also addresses how EA resources that focus on developing and deploying EA artifacts enable firms' dynamic capabilities. This paper embraces a deductive approach and tests the model's hypotheses using a cross-sectional dataset that contains responses from 299 CIO's, IT managers, and lead architects. Outcomes of this study extend previous empirical studies and show that dynamic enterprise architecture capabilities positively enhance firms' process innovation and business-IT alignment. These mediating forces are both positively associated with organizational benefits. The firms' EA resources and EA deployment practices are essential in cultivating dynamic enterprise architecture capabilities. This study's findings might explain why firms still encounter organizational and externally imposed obstacles with realizing EA's intended business outcomes and advance our understanding of how to efficiently delineate dynamic enterprise architecture capabilities in delivering benefits to the organization.

\section{Concluding Comments}

This special issue's contributions showcase the need for research on EA's role in digital transformation from various complementary perspectives, methods, and approaches. We believe that research on EA's value in digital transformation will further accelerate in the coming years. We hope that this special issue provokes IS and EA scholars to take on the next efforts to investigate EA's role in digital transformations and serves as a guide to embracing new multidisciplinary research avenues and perspectives. See Table 1. 
Table 1. References of the articles in this Special Issue.

\begin{tabular}{ll}
\hline Article & Reference \\
\hline I. & Gerber, A.; le Roux, P.; van der Merwe, A. Enterprise Architecture as Explanatory Information Systems Theory for Understanding \\
II. & Small-and Medium-Sized Enterprise Growth. Sustainability 2020, 12, 8517. \\
III. & Saleem, F.; Fakieh, B. Enterprise Architecture and Organizational Benefits: A Case Study. Sustainability 2020, 12, 8237. \\
IV. & Alwadain, A. Enterprise Architecture: A Business Value Realization Model. Sustainability 2020, 12, 8485. \\
& Dorofeev, A.; Altukhova, N.; Filippova, N.; Pashkova, T.; Ponomarev, M. Development of Transportation Management System with the \\
V. & Use of Ontological and Architectural Approaches to Ensure Trucking Reliability. Sustainability 2020, 12, 8504. \\
& Vyn de Wetering, R.; Kurnia, S.; Kotusev, S. The Effect of Enterprise Architecture Deployment Practices on Organizational Benefits: A \\
\hline
\end{tabular}

Author Contributions: Conceptualization, R.v.d.W.; Investigation, R.v.d.W.; Resources, S.K. (Sherah Kurnia); Supervision, R.v.d.W.; Visualization, R.v.d.W. and S.K. (Sherah Kurnia); Writing-original draft, R.v.d.W.; Writing-review \& editing, S.K. (Sherah Kurnia) and S.K. (Svyatoslav Kotusev). All authors have read and agreed to the published version of the manuscript.

Funding: This research received no external funding.

Acknowledgments: We are grateful for the opportunity to edit this Special Issue for the Sustainability Journal. The Special Issue would not have been possible without the Special Issue editorial review board and reviewers' tremendous help. We are also grateful to the scholars that contributed to this issue.

Conflicts of Interest: The authors declare no conflict of interest.

\section{References}

1. Bharadwaj, A.; El Sawy, O.; Pavlou, P.; Venkatraman, N. Digital business strategy: Toward a next generation of insights. MIS Q. 2013, 37, 471-482. [CrossRef]

2. Eggers, J.; Park, K.F. Incumbent adaptation to technological change: The past, present, and future of research on heterogeneous incumbent response. Acad. Manag. Ann. 2018, 12, 357-389. [CrossRef]

3. Aral, S.; Weill, P. IT assets, organizational capabilities, and firm performance: How resource allocations and organizational differences explain performance variation. Organ. Sci. 2007, 18, 763-780. [CrossRef]

4. Hitt, L.M.; Brynjolfsson, E. Productivity, business profitability, and consumer surplus: Three different measures of information technology value. MIS Q. 1996, 20, 121-142. [CrossRef]

5. $\quad$ Ross, J.W.; Weill, P.; Robertson, D. Enterprise Architecture as Strategy: Creating a Foundation for Business Execution; Harvard Business Press: Brighton, MA, USA, 2006.

6. Shanks, G.; Gloet, M.; Someh, I.A.; Frampton, K.; Tamm, T. Achieving benefits with enterprise architecture. J. Strateg. Inf. Syst. 2018, 27, 139-156. [CrossRef]

7. Lee, S.; Oh, S.W.; Nam, K. Transformational and transactional factors for the successful implementation of enterprise architecture in public sector. Sustainability 2016, 8, 456. [CrossRef]

8. Hazen, B.T.; Bradley, R.V.; Bell, J.E.; In, J.; Byrd, T.A. Enterprise architecture: A competence-based approach to achieving agility and firm performance. Management 2017, 193, 566-577. [CrossRef]

9. Bradley, R.V.; Pratt, R.M.; Byrd, T.A.; Outlay, C.N.; Wynn, J.; Donald, E. Enterprise architecture, IT effectiveness and the mediating role of IT alignment in US hospitals. Inf. Syst. J. 2012, 22, 97-127. [CrossRef]

10. Ross, J.W.; Beath, C.M.; Mocker, M. Designed for Digital: How to Architect Your Business for Sustained Success; Mit Press: Cambridge, MA, USA, 2019.

11. Nam, K.; Oh, S.W.; Kim, S.K.; Goo, J.; Khan, M.S. Dynamics of enterprise architecture in the Korean public sector: Transformational change vs. transactional change. Sustainability 2016, 8, 1074. [CrossRef]

12. Allega, P. Hype Cycle for Enterprise Architecture; Gartner(G00467339). 2020. Available online: https://www.gartner.com/en/ documents /3989875/hype-cycle-for-enterprise-architecture-2020 (accessed on 19 February 2021).

13. Van de Wetering, R. Enterprise Architecture Resources, Dynamic Capabilities, and their Pathways to Operational Value. In Proceedings of the 40th International Conference on Information Systems, Munich, Germany, 15-18 December 2019.

14. Van de Wetering, R. Dynamic Enterprise Architecture Capabilities and Organizational Benefits: An empirical mediation study. In Proceedings of the 28th European Conference on Information Systems, Marrakech, Morocco, 15-17 June 2020.

15. Pattij, M.; Van de Wetering, R.; Kusters, R.J. Improving Agility Through Enterprise Architecture Management: The Mediating Role of Aligning Business and IT. In Proceedings of the 25th Americas Conference on Information Systems (AMCIS), Salt Lake City, UT, USA, 12-16 August 2020. 